\title{
On generalized absolute Cesàro summability factors
}

\section{A Nihal Tuncer*}

\section{"Correspondence:} ntuncer@erciyes.edu.tr

Department of Mathematics, Erciyes University, Kayseri, 38039, Turkey

\section{Abstract}

In this paper, a known theorem dealing with $|C, \alpha, \gamma ; \delta|_{k}$ summability factors has been generalized for $|C, \alpha, \beta, \gamma ; \delta|_{k}$ summability factors. Some results have also been obtained.

MSC: 40D15; 40F05; 40G99

Keywords: Hölder's inequality; quasi-monotone sequence; summability factors

\section{Introduction}

A sequence $\left(b_{n}\right)$ of positive numbers is said to be quasi-monotone if $n \Delta b_{n} \geq-\rho b_{n}$ for some $\rho>0$ and is said to be $\delta$-quasi-monotone, if $b_{n} \rightarrow 0, b_{n}>0$ ultimately and $\Delta b_{n} \geq-\delta_{n}$, where $\left(\delta_{n}\right)$ is a sequence of positive numbers (see [1]). Let $\sum a_{n}$ be a given infinite series with partial sums $\left(s_{n}\right)$. We denote by $u_{n}^{\alpha, \beta}$ and $t_{n}^{\alpha, \beta}$ the $n$th Cesàro means of order $(\alpha, \beta)$, with $\alpha+\beta>-1$, of the sequences $\left(s_{n}\right)$ and $\left(n a_{n}\right)$, respectively, that is (see [2]),

$$
\begin{aligned}
& u_{n}^{\alpha, \beta}=\frac{1}{A_{n}^{\alpha+\beta}} \sum_{v=0}^{n} A_{n-v}^{\alpha-1} A_{v}^{\beta} s_{v}, \\
& t_{n}^{\alpha, \beta}=\frac{1}{A_{n}^{\alpha+\beta}} \sum_{v=1}^{n} A_{n-v}^{\alpha-1} A_{v}^{\beta} v a_{v},
\end{aligned}
$$

where

$$
A_{n}^{\alpha+\beta}=O\left(n^{\alpha+\beta}\right), \quad \alpha+\beta>-1, \quad A_{0}^{\alpha+\beta}=1 \quad \text { and } \quad A_{-n}^{\alpha+\beta}=0 \quad \text { for } n>0 .
$$

The series $\sum a_{n}$ is said to be summable $|C, \alpha, \beta|_{k}, k \geq 1$ and $\alpha+\beta>-1$, if (see [3])

$$
\sum_{n=1}^{\infty} n^{k-1}\left|u_{n}^{\alpha, \beta}-u_{n-1}^{\alpha, \beta}\right|^{k}<\infty .
$$

Since $t_{n}^{\alpha, \beta}=n\left(u_{n}^{\alpha, \beta}-u_{n-1}^{\alpha, \beta}\right)$ (see [3]), condition (4) can also be written as

$$
\sum_{n=1}^{\infty} \frac{1}{n}\left|t_{n}^{\alpha, \beta}\right|^{k}<\infty
$$


The series $\sum a_{n}$ is said to be summable $|C, \alpha, \beta, \gamma ; \delta|_{k}, k \geq 1, \alpha+\beta>-1, \delta \geq 0$ and $\gamma$ is a real number, if (see [4])

$$
\sum_{n=1}^{\infty} n^{\gamma(\delta k+k-1)}\left|u_{n}^{\alpha, \beta}-u_{n-1}^{\alpha, \beta}\right|^{k}=\sum_{n=1}^{\infty} n^{\gamma(\delta k+k-1)-k}\left|t_{n}^{\alpha, \beta}\right|^{k}<\infty
$$

If we take $\beta=0$, then $|C, \alpha, \beta, \gamma ; \delta|_{k}$ summability reduces to $|C, \alpha, \gamma ; \delta|_{k}$ summability (see [5]).

\section{Known result}

In [6], we have proved the following theorem dealing with $|C, \alpha, \gamma ; \delta|_{k}$ summability factors of infinite series.

Theorem A Let $k \geq 1,0 \leq \delta<\alpha \leq 1$, and $\gamma$ be a real number such that $-\gamma(\delta k+k-1)+$ $(\alpha+1) k>1$. Suppose that there exists a sequence of numbers $\left(B_{n}\right)$ such that it is $\delta$-quasimonotone with $\left|\Delta \lambda_{n}\right| \leq\left|B_{n}\right|, \lambda_{n} \rightarrow 0$ as $n \rightarrow \infty, \sum_{n=1}^{\infty} n \delta_{n} \log n<\infty$ and $\sum_{n=1}^{\infty} n B_{n} \log n$ is convergent. If the sequence $\left(w_{n}^{\alpha}\right)$ defined by (see [7])

$$
\begin{aligned}
& w_{n}^{\alpha}=\left|t_{n}^{\alpha}\right|, \quad \alpha=1, \\
& w_{n}^{\alpha}=\max _{1 \leq v \leq n}\left|t_{v}^{\alpha}\right|, \quad 0<\alpha<1,
\end{aligned}
$$

satisfies the condition

$$
\sum_{n=1}^{m} n^{\gamma(\delta k+k-1)-k}\left(w_{n}^{\alpha}\right)^{k}=O(\log m) \quad \text { as } m \rightarrow \infty
$$

then the series $\sum a_{n} \lambda_{n}$ is summable $|C, \alpha, \gamma ; \delta|_{k}$.

\section{The main result}

The aim of this paper is to generalize Theorem $\mathrm{A}$ for $|C, \alpha, \beta, \gamma ; \delta|_{k}$ summability. We shall prove the following theorem.

Theorem Let $k \geq 1,0 \leq \delta<\alpha \leq 1$, and $\gamma$ be a real number such that $(\alpha+\beta+1-\gamma(\delta+$ $1)) k>1$, and let there be sequences $\left(B_{n}\right)$ and $\left(\lambda_{n}\right)$ such that the conditions of Theorem A are satisfied. If the sequence $\left(w_{n}^{\alpha, \beta}\right)$ defined by

$$
\begin{aligned}
& w_{n}^{\alpha, \beta}=\left|t_{n}^{\alpha, \beta}\right|, \quad \alpha=1, \beta>-1, \\
& w_{n}^{\alpha, \beta}=\max _{1 \leq v \leq n}\left|t_{v}^{\alpha, \beta}\right|, \quad 0<\alpha<1, \beta>-1,
\end{aligned}
$$

satisfies the condition

$$
\sum_{n=1}^{m} n^{\gamma(\delta k+k-1)-k}\left(w_{n}^{\alpha, \beta}\right)^{k}=O(\log m) \quad \text { as } m \rightarrow \infty,
$$

then the series $\sum a_{n} \lambda_{n}$ is summable $|C, \alpha, \beta, \gamma ; \delta|_{k}$. It should be noted that if we take $\beta=0$, then we get Theorem A. 
We need the following lemmas for the proof of our theorem.

Lemma 1 ([8]) Under the conditions on $\left(B_{n}\right)$, as taken in the statement of the theorem, we have the following:

$$
\begin{aligned}
& n B_{n} \log n=O(1), \\
& \sum_{n=1}^{\infty} n \log n\left|\Delta B_{n}\right|<\infty .
\end{aligned}
$$

Lemma 2 ([9]) If $0<\alpha \leq 1, \beta>-1$, and $1 \leq v \leq n$, then

$$
\left|\sum_{p=0}^{v} A_{n-p}^{\alpha-1} A_{p}^{\beta} a_{p}\right| \leq \max _{1 \leq m \leq \nu}\left|\sum_{p=0}^{m} A_{m-p}^{\alpha-1} A_{p}^{\beta} a_{p}\right| .
$$

\section{Proof of the theorem}

Let $\left(T_{n}^{\alpha, \beta}\right)$ be the $n$th $(C, \alpha, \beta)$ mean of the sequence $\left(n a_{n} \lambda_{n}\right)$. Then by (2), we have

$$
T_{n}^{\alpha, \beta}=\frac{1}{A_{n}^{\alpha+\beta}} \sum_{v=1}^{n} A_{n-v}^{\alpha-1} A_{v}^{\beta} v a_{v} \lambda_{v} .
$$

Firstly applying Abel's transformation and then using Lemma 2, we have that

$$
\begin{aligned}
T_{n}^{\alpha, \beta}= & \frac{1}{A_{n}^{\alpha+\beta}} \sum_{v=1}^{n-1} \Delta \lambda_{v} \sum_{p=1}^{v} A_{n-p}^{\alpha-1} A_{p}^{\beta} p a_{p}+\frac{\lambda_{n}}{A_{n}^{\alpha+\beta}} \sum_{v=1}^{n} A_{n-v}^{\alpha-1} A_{v}^{\beta} v a_{v}, \\
\left|T_{n}^{\alpha, \beta}\right| & \leq \frac{1}{A_{n}^{\alpha+\beta}} \sum_{v=1}^{n-1}\left|\Delta \lambda_{v}\right|\left|\sum_{p=1}^{v} A_{n-p}^{\alpha-1} A_{p}^{\beta} p a_{p}\right|+\frac{\left|\lambda_{n}\right|}{A_{n}^{\alpha+\beta}}\left|\sum_{v=1}^{n} A_{n-v}^{\alpha-1} A_{v}^{\beta} v a_{v}\right| \\
& \leq \frac{1}{A_{n}^{\alpha+\beta}} \sum_{v=1}^{n-1} A_{v}^{\alpha} A_{v}^{\beta} w_{v}^{\alpha, \beta}\left|\Delta \lambda_{v}\right|+\left|\lambda_{n}\right| w_{n}^{\alpha, \beta}=T_{n, 1}^{\alpha, \beta}+T_{n, 2}^{\alpha, \beta}, \quad \text { say }
\end{aligned}
$$

since

$$
\left|T_{n, 1}^{\alpha, \beta}+T_{n, 2}^{\alpha, \beta}\right|^{k} \leq 2^{k}\left(\left|T_{n, 1}^{\alpha, \beta}\right|^{k}+\left|T_{n, 2}^{\alpha, \beta}\right|^{k}\right) .
$$

In order to complete the proof of the theorem, by (6), it is sufficient to show that for $r=1,2$,

$$
\sum_{n=1}^{\infty} n^{\gamma(\delta k+k-1)-k}\left|T_{n, r}^{\alpha, \beta}\right|^{k}<\infty
$$

Whenever $k>1$, we can apply Hölder's inequality with indices $k$ and $k^{\prime}$, where $\frac{1}{k}+\frac{1}{k^{\prime}}=1$, we get that

$$
\begin{aligned}
& \sum_{n=2}^{m+1} n^{\gamma(\delta k+k-1)-k}\left|T_{n, 1}^{\alpha, \beta}\right|^{k} \\
& \quad \leq \sum_{n=2}^{m+1} n^{\gamma(\delta k+k-1)-k}\left|\frac{1}{A_{n}^{\alpha+\beta}} \sum_{v=1}^{n-1} A_{v}^{\alpha} A_{v}^{\beta} w_{v}^{\alpha, \beta} \Delta \lambda_{v}\right|^{k}
\end{aligned}
$$




$$
\begin{aligned}
& =O(1) \sum_{n=2}^{m+1} \frac{1}{n^{(\alpha+\beta+1-\gamma(\delta+1)) k}}\left\{\sum_{v=1}^{n-1} v^{\alpha k} v^{\beta k}\left|\Delta \lambda_{v}\right|\left(w_{v}^{\alpha, \beta}\right)^{k}\right\}\left\{\sum_{v=1}^{n-1}\left|\Delta \lambda_{v}\right|\right\}^{k-1} \\
& =O(1) \sum_{n=2}^{m+1} \frac{1}{n^{(\alpha+\beta+1-\gamma(\delta+1)) k}}\left\{\sum_{v=1}^{n-1} v^{\alpha k} v^{\beta k}\left|B_{v}\right|\left(w_{v}^{\alpha, \beta}\right)^{k}\right\}\left\{\sum_{v=1}^{n-1}\left|B_{v}\right|\right\}^{k-1} \\
& =O(1) \sum_{v=1}^{m} v^{(\alpha+\beta) k}\left|B_{v}\right|\left(w_{v}^{\alpha, \beta}\right)^{k} \sum_{n=v+1}^{m+1} \frac{1}{n^{(\alpha+\beta+1-\gamma(\delta+1)) k}} \\
& =O(1) \sum_{v=1}^{m} v^{(\alpha+\beta) k}\left|B_{v}\right|\left(w_{v}^{\alpha, \beta}\right)^{k} \int_{v}^{\infty} \frac{d x}{x^{(\alpha+\beta+1-\gamma(\delta+1)) k}} \\
& =O(1) \sum_{v=1}^{m}\left|B_{v}\right| v^{\gamma(\delta k+k-1)-k+1}\left(w_{v}^{\alpha, \beta}\right)^{k} \\
& =O(1) \sum_{v=1}^{m} v\left|B_{v}\right| v^{\gamma(\delta k+k-1)-k}\left(w_{v}^{\alpha, \beta}\right)^{k} \\
& =O(1) \sum_{v=1}^{m-1}\left|\Delta\left(v\left|B_{v}\right|\right)\right| \sum_{p=1}^{v} p^{\gamma(\delta k+k-1)-k}\left(w_{p}^{\alpha, \beta}\right)^{k}+O(1) m\left|B_{m}\right| \sum_{v=1}^{m} v^{\gamma(\delta k+k-1)-k}\left(w_{v}^{\alpha, \beta}\right)^{k} \\
& =O(1) \sum_{v=1}^{m-1}\left|\Delta\left(v\left|B_{v}\right|\right)\right| \log v+O(1) m\left|B_{m}\right| \log m \\
& =O(1) \sum_{v=1}^{m-1} v\left|\Delta B_{v}\right| \log v+O(1) \sum_{v=1}^{m-1}\left|B_{v+1}\right| \log v+O(1) m\left|B_{m}\right| \log m \\
& =O(1) \operatorname{as}^{m} m \rightarrow \infty,
\end{aligned}
$$

in view of the hypotheses of the theorem and Lemma 1. Similarly, we have that

$$
\begin{aligned}
\sum_{n=2}^{m+1} n^{\gamma(\delta k+k-1)-k}\left|T_{n, 2}^{\alpha, \beta}\right|^{k}= & O(1) \sum_{n=1}^{m}\left|\lambda_{n}\right| n^{\gamma(\delta k+k-1)-k}\left(w_{n}^{\alpha, \beta}\right)^{k} \\
= & O(1) \sum_{n=1}^{m-1}\left|\Delta \lambda_{n}\right| \sum_{v=1}^{n} v^{\gamma(\delta k+k-1)-k}\left(w_{v}^{\alpha, \beta}\right)^{k} \\
& +O(1)\left|\lambda_{m}\right| \sum_{v=1}^{m} v^{\gamma(\delta k+k-1)-k}\left(w_{v}^{\alpha, \beta}\right)^{k} \\
= & O(1) \sum_{n=1}^{m-1}\left|\Delta \lambda_{n}\right| \log n+O(1)\left|\lambda_{m}\right| \log m \\
= & O(1) \sum_{n=1}^{m-1}\left|B_{n}\right| \log n+O(1)\left|\lambda_{m}\right| \log m \\
= & O(1) \quad \text { as } m \rightarrow \infty
\end{aligned}
$$

by virtue of the hypotheses of the theorem and Lemma 1 . Therefore, by (6), we get that for $r=1,2$,

$$
\sum_{n=1}^{\infty} n^{\gamma(\delta k+k-1)-k}\left|T_{n, r}^{\alpha, \beta}\right|^{k}<\infty .
$$


This completes the proof of the theorem. If we take $\delta=0$ and $\gamma=1$, then we get a result for $|C, \alpha, \beta|_{k}$ summability factors. Also, if we take $\beta=0, \delta=0$, and $\alpha=1$, then we get a result for $|C, 1|_{k}$ summability.

\section{Competing interests}

The author declares that they have no competing interests.

Received: 23 December 2011 Accepted: 17 October 2012 Published: 30 October 2012

\section{References}

1. Boas, RP: Quasi-positive sequences and trigonometric series. Proc. Lond. Math. Soc. 14, $38-46$ (1965)

2. Borwein, D: Theorems on some methods of summability. Q. J. Math. 9, 310-316 (1958)

3. Das, G: A Tauberian theorem for absolute summability. Proc. Camb. Philos. Soc. 67, 321-326 (1970)

4. Bor, H: On generalized absolute Cesàro summability. Pac. J. Appl. Math. 2, 35-40 (2009)

5. Tuncer, AN: On generalized absolute Cesàro summability factors. Ann. Pol. Math. 78, 25-29 (2002)

6. Tuncer, AN: Factors for absolute Cesàro summability. Tamkang J. Math. 32, 21-25 (2001)

7. Pati, T: The summability factors of infinite series. Duke Math. J. 21, 271-284 (1954)

8. Mazhar, SM: On a generalized quasi-convex sequence and its applications. Indian J. Pure Appl. Math. 8, 784-790 (1977)

9. Bor, H: On a new application of quasi power increasing sequences. Proc. Est. Acad. Sci. 57, 205-209 (2008)

doi:10.1186/1029-242X-2012-253

Cite this article as: Tuncer: On generalized absolute Cesàro summability factors. Journal of Inequalities and

Applications 2012 2012:253.

\section{Submit your manuscript to a SpringerOpen ${ }^{\circ}$ journal and benefit from:}

- Convenient online submission

- Rigorous peer review

- Immediate publication on acceptance

- Open access: articles freely available online

- High visibility within the field

- Retaining the copyright to your article 